\title{
Online modelling of water distribution systems: a UK case study
}

\author{
J. Machell, S. R. Mounce, and J. B. Boxall \\ Pennine Water Group, Department of Civil and Structural Engineering, University of Sheffield, Sheffield, UK
}

Received: 11 December 2009 - Published in Drink. Water Eng. Sci. Discuss.: 23 December 2009

Revised: 12 March 2010 - Accepted: 24 March 2010 - Published: 30 March 2010

\begin{abstract}
Hydraulic simulation models of water distribution networks are routinely used for operational investigations and network design purposes. However, their full potential is often never realised because, in the majority of cases, they have been calibrated with data collected manually from the field during a single historic time period and, as such, reflect the network operational conditions that were prevalent at that time, and they are then applied as part of a reactive, desktop investigation. In order to use a hydraulic model to assist proactive distribution network management its element asset information must be up to date and it should be able to access current network information to drive simulations. Historically this advance has been restricted by the high cost of collecting and transferring the necessary field measurements. However, recent innovation and cost reductions associated with data transfer is resulting in collection of data from increasing numbers of sensors in water supply systems, and automatic transfer of the data to point of use. This means engineers potentially have access to a constant stream of current network data that enables a new era of "on-line" modelling that can be used to continually assess standards of service compliance for pressure and reduce the impact of network events, such as mains bursts, on customers. A case study is presented here that shows how an online modelling system can give timely warning of changes from normal network operation, providing capacity to minimise customer impact.
\end{abstract}

\section{Introduction}

The day to day operation of a water supply system is managed by skilled staff that use their experience and expert judgement to adjust control elements such as pumps and valves to ensure customer demands on the systems are met (regulatory compliance), and to minimise energy costs. As water use tends to follow repeatable patterns the operators understand what is required for normal operation but, when an unplanned event such as a burst main occurs, they often have to respond in a reactive manner, usually with scant information provided by contact from customers already impacted by the event. Because water companies are constantly striving to improve standards of service delivery and increase customer satisfaction, there is a need to shift from this reactive approach to a more proactive management strategy. One of the tools that can be used to help accomplish this shift is an online hydraulic network simulation model.
Network simulation software provides the capability to mathematically replicate the nonlinear dynamics of a water distribution network by solving the governing set of quasisteady state hydraulic equations that include conservation of mass and conservation of energy elements. Various numerical methods have been utilised for solving these equations and in recent years the combination of the nodal heads and the Newton-Raphson algorithm has been the most frequently used procedure for solving water networks (Powell et al., 1988). Numerical simulation for online hydraulic modelling using state estimation has been receiving increased attention (Sterling and Bargiela, 1984). For example it has been shown that the loop equations method is a suitable framework for the inclusion of pressure-controlling elements without specifying the operational state of the network (Andersen and Powell, 1999). 
Hydraulic simulation software is in widespread use within the water industry for strategic supply analysis, design of control strategies, network extensions, and maintenance planning. A number of off the shelf packages are available that allow tailor made simulation models to be constructed for a water companies specific requirements. Popular packages include EPANET (US Environmental Protection Agency), AQUIS (7T), Infoworks (Wallingford software), and SynerGEE (Advantica). These simulation software packages enable the implementation of mathematical models of a water distribution networks that combine the physical laws governing the networks with the equations that relate pressure and flow for each operational element.

The design of distribution networks using these software packages has developed from trial and error to, more recently, the use of various forms of optimization, including genetic algorithms (e.g. Dandy et al., 1996). Tools of this type are offline in nature as, although very efficient, the computational burden is often large. Real time, near optimal control using hydraulic modelling is still somewhat impractical for large water distribution networks because of the computational burden such optimization imposes, and alternative approaches are being developed. Bhattacharya et al. (2003) proposed an ANN (Artificial Neural Network) with reinforcement learning which could learn to replicate the optimal control strategy (based on capturing operator experience). Rao and Salomons (2007) developed a GA (Genetic Algorithm) and an ANN model for capturing the knowledge base of an EPANET model and consequently producing a near optimal solution for control settings under dynamic conditions (with $1 \mathrm{~h}$ SCADA updates).

For any hydraulic model to be useful to operators it should contain accurate element physical characteristic information and be calibrated, by adjusting the model variable parameters until field measurement and model simulation results are in reasonable agreement, so that it accurately reflects the true operational characteristics of the network. This process generally requires a considerable amount of data, for example, around 14 days of measured flow and pressure from the network, which has traditionally been collected manually, and manipulation of model variables. Kapelan et al. (2000) reviews calibration models. Koppel and Vassiljev (2008) illustrate the calibration problem with a case study.

Some preliminary efforts have been made to link hydraulic simulation models with on-line data from telemetry systems for operational purposes (Skipworth et al., 1999; Orr et al., 1999). The idea was to associate a well calibrated network hydraulic model with a SCADA (Supervisory Control and Data Acquisition) system that populated a database with near real time measurements of flow and pressure from strategic points in the network that could be accessed by a simulation model. However, data was transmitted via telephone lines and wired modems which frequently could not connect to some of the data loggers in the network. The system was slow and suffered data corruption problems. Also, be- cause every data logger had to be contacted individually via a telephone call to obtain a single, current reading, the time required to gather data from all the loggers was significant thereby introducing data "skew" that could affect simulation results.

This paper describes an online hydraulic simulation model and demonstrates its potential by showing details of a flow event detected in one case study network DMA (Distribution Management Areas). It is shown that a sufficiently accurate on-line model may be used to detect hydraulic events such as bursts and if correctly configured, such a model can also detect illegal hydrant or valve operations, and monitor standards of service levels for pressure.

\section{Conventional modelling of distribution networks}

A conventional distribution network simulation model is comprised of static asset information including pipe lengths, diameters, connectivity, and network topography; and information about dynamic parameters, for example, the distribution of demand, and elements such as pumps, reservoirs and valves. Most contemporary modelling packages in current use utilise models that include every main down to the level of, but not including, customer service pipes. Standard daily time-varying demand relationships are estimated for different customer types, and these are summed and allocated at pipe junctions (nodes) according to the distribution of customers. The models are normally used to simulate flows and pressures over a fixed (normalised data from a specific date) $24 \mathrm{~h}$ period which provides enough information to be suitable for purpose. Since their advent, these complex calibrated models have predominantly been used for leakage management and control, specifically for splitting distribution networks into discrete DMAs, the design of pressure reduction schemes, and the location of substantial leaks during model calibration exercises.

\section{On-line modelling of distribution networks}

An online simulation model requires the same information as a conventional model, but uses current, near real time data for boundary conditions; the simulation initial parameter values at key locations. Rather than simulate a fixed $24 \mathrm{~h}$ period as conventional models, an online model is most useful to the operator if it provides the latest period of operation and ideally a look ahead for likely performance in the next few hours, based on extrapolation of current performance with respect to the original offline model. An online model can calculate the current network status and status for the next few time steps based on current measurements and the inbuilt predicted demand patterns. Complex models can have hundreds of measurements imposed as simulation boundary conditions at the inlets and outlets of DMAs, service reservoirs and pumping stations, in fact, anywhere that flow or pressure 


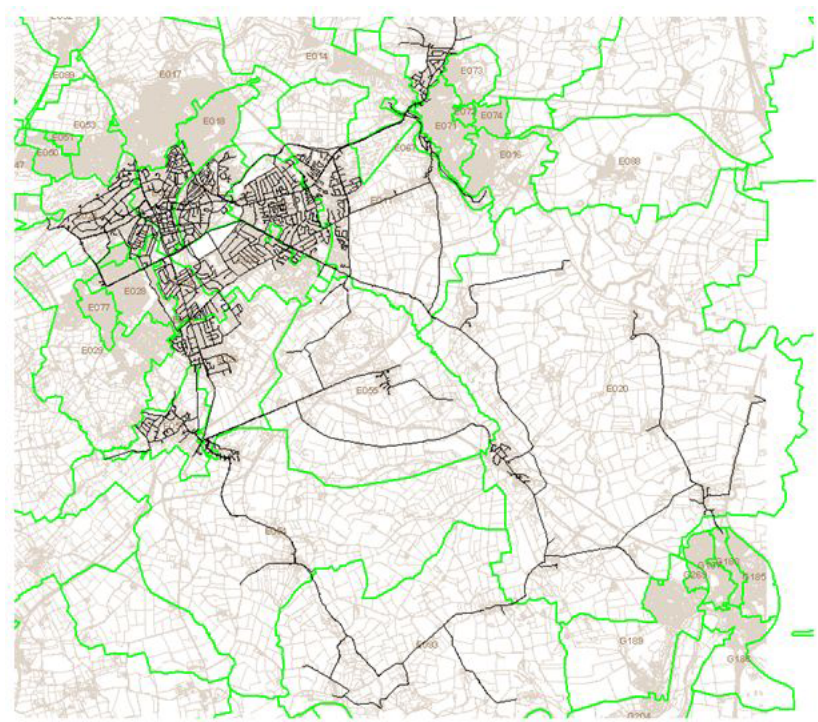

Figure 1. Online model geographical extent showing all pipes, background map and DMA boundaries.

is measured in the real network. In order to simulate the current hydraulic state of a network, demand constraints within the model are relaxed and their adjustment allows for a solution to be calculated that matches the measured hydraulic conditions.

\section{The On-line model}

This paper presents an online model using the Aquis 1.47 (7T) software. Aquis is a water network simulation platform used in daily operation in 1500 cities worldwide, and is an industry standard modelling package in the UK (http: //www.7t.dk/aquis/). The model is driven by near real-time flow and pressure data gathered using GPRS communication technologies that downloaded data with a $15 \mathrm{~min}$ sample interval every $30 \mathrm{~min}$. This GPRS technology includes accurate time stamping, irrespective of any intermittent communication errors, that ensures no data skewing occurs across measurement sites. The online model can run every $30 \mathrm{~min}$ on 15 min sampled data and takes only a minute or two to run one real-time cycle on an average spec desktop PC. Even when scaling up to the larger 16 DMA model this frequency of recalculation described is feasible. The full area covered by the online hydraulic model includes a large area of a town of 160000 people (approximately 25500 properties) and is supplied with 77 near real time measurements. It comprises 16 DMAs, 4 service reservoirs, 2 pumping stations, 9081 pipes and 8571 nodes. Figure 1 shows the geographical extent of the model.

\subsection{Model construction and calibration}

A conventional network simulation model calculates a hydraulic solution under a set of constraints which correspond to an estimated distribution of demand for an idealized $24 \mathrm{~h}$ period. Calibration of such models is usually to averaged pressure data from a relatively short period and with limited coverage. It is possible for idealised $24 \mathrm{~h}$ models to provide apparently sensible, but not wholly accurate, solutions for non-representative model constraints. For an on-line simulation, with numerous hydraulic flow and pressure boundary conditions imposed, these constraints are relaxed, leading to the requirement for a higher degree of accuracy both in the model calibration procedure and the on-line flow and pressure measurements. Hence, the first requirement for an on-line model is for an accurate representation of the network and a well calibrated conventional hydraulic model. For the work reported here the calibrated conventional offline model was built by consultants on behalf of the water company. Once a suitable conventional hydraulic model has been produced, it is necessary to connect it for on-line operation. This is achieved by a SCADA system to provide the flow and pressure data for simulation of boundary conditions, and a data management system that checks data status and pre-processes it in preparation for use in the model.

\subsection{Data route and interfacing}

Field data from the logger and GPRS system was automatically forwarded via FTP to a server at the University of Sheffield and a windows service automatically copied the updated files to a file store. DataManager, an online database application for configuring, pre-processing and administering the raw data for use with AQUIS, accessed the stored files and transferred them into an integral MS Access database where all flow and pressure time series used by the online model are stored. The online model was linked to the database and a data pre-processor then checked the raw data against defined parameters for missing or corrupt data. Where raw data was missing or corrupt (as specified by being outside predefined limits), a data emulation module can be used with one of three options selected as configured by operator specified rules. Firstly, it can be replaced with default value (which remains constant). Secondly, it can be replaced by an average over previous values (those values not including faulty data or emulated data). Finally, a reference tag value can be used in which measurements are substituted by the converted measurement from another tag. On completion of the pre-processing, the data was imposed as boundary conditions for simulations at the appropriate nodes/pipes within the model. 


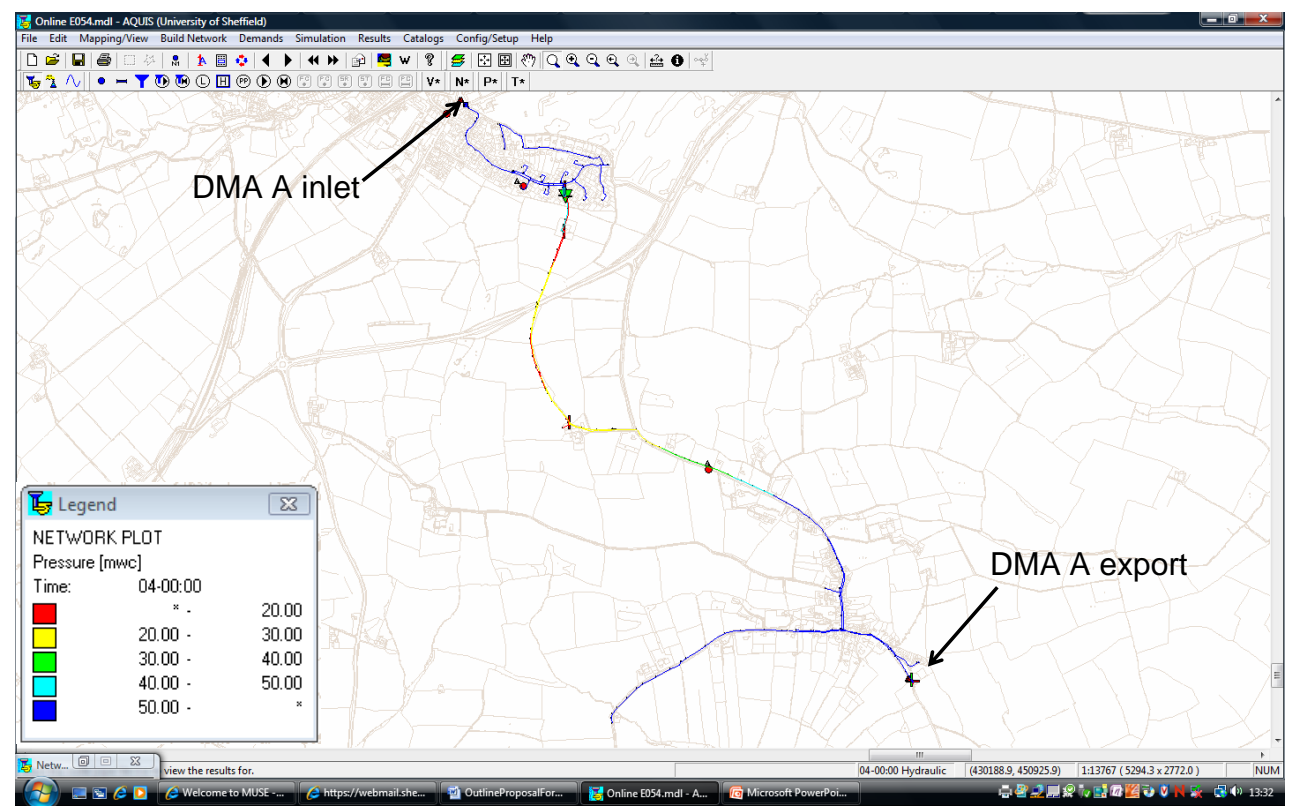

Figure 2. Example DMA network plot with normal pressure overlay.

\subsection{Example event}

As stated above an online model has been successfully created for a significant network area. In order to tangibly demonstrate the capabilities of the online model attention is focused on a single DMA where an unusual event is known to have occurred.

The DMA used for the selected example is a semi rural DMA with customers concentrated at the north end. The DMA selected consists of 269 domestic and 18 commercial properties. It has pipes made of asbestos, cement, cast and ductile iron and polyethylene, diameters from 50 to $300 \mathrm{~mm}$. For the purposes of the results reported here, a single DMA model was extracted from the full online model with real time data for the DMA inlet and export flows streamed into the model and imposed as simulation boundary conditions using DataManager. The model was then checked to confirm that under "normal" conditions the online simulation results were comparable to those produced by the conventional hydraulic model, at the time of calibration. This confirmed that under normal operational flow conditions the online model produced output that accurately reflected the actual pressure conditions found in the real network. Figure 2 shows a network plot with pressure overlay, and the locations of the imposed flows under normal operational conditions.

During a period of operations a fire hydrant was opened producing a flow to waste of approximately $31 / \mathrm{s}$, this was confirmed by an Artificial Intelligence based alert system running concurrently (Mounce and Boxall, 2009). Figure 3 illustrates the flow and pressure changes (for the DMA export) that occurred during the event and some details of the timings.

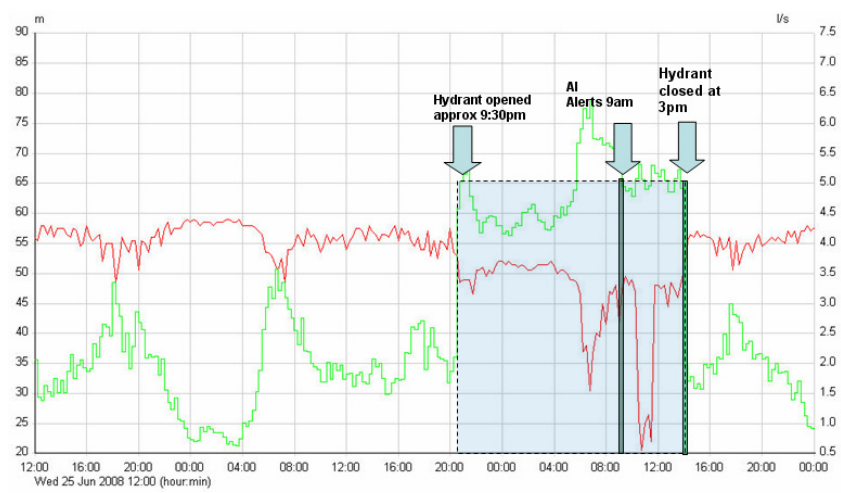

Figure 3. Details of event as reported by Artificial Intelligence based alert system.

Figure 3 shows that increased flow and decreased pressure was measured by the instruments associated with the selected DMA, this is interpreted to infer that a hydrant was opened at 21:30, 26 June 2008. The hydrant opening was detected at 9 a.m. by the AI system the following morning and estimated the event flow to be $2.8 \mathrm{l} / \mathrm{s}$ (the AI system operates on a twelve hour window). The alert was noted in the water company control room and a field operative sent to investigate. The hydrant was subsequently found, in the DMA downstream of the one presented here, and closed at 15:00 $\mathrm{h}$.

In Fig. 3 a sudden pressure drop at 11 a.m. (more than $20 \mathrm{~m}$ ) can also be observed. There is no corresponding increase in flow (unlike the flow increase and pressure drop at 7 a.m. at peak demand). This is reflection of using real field 


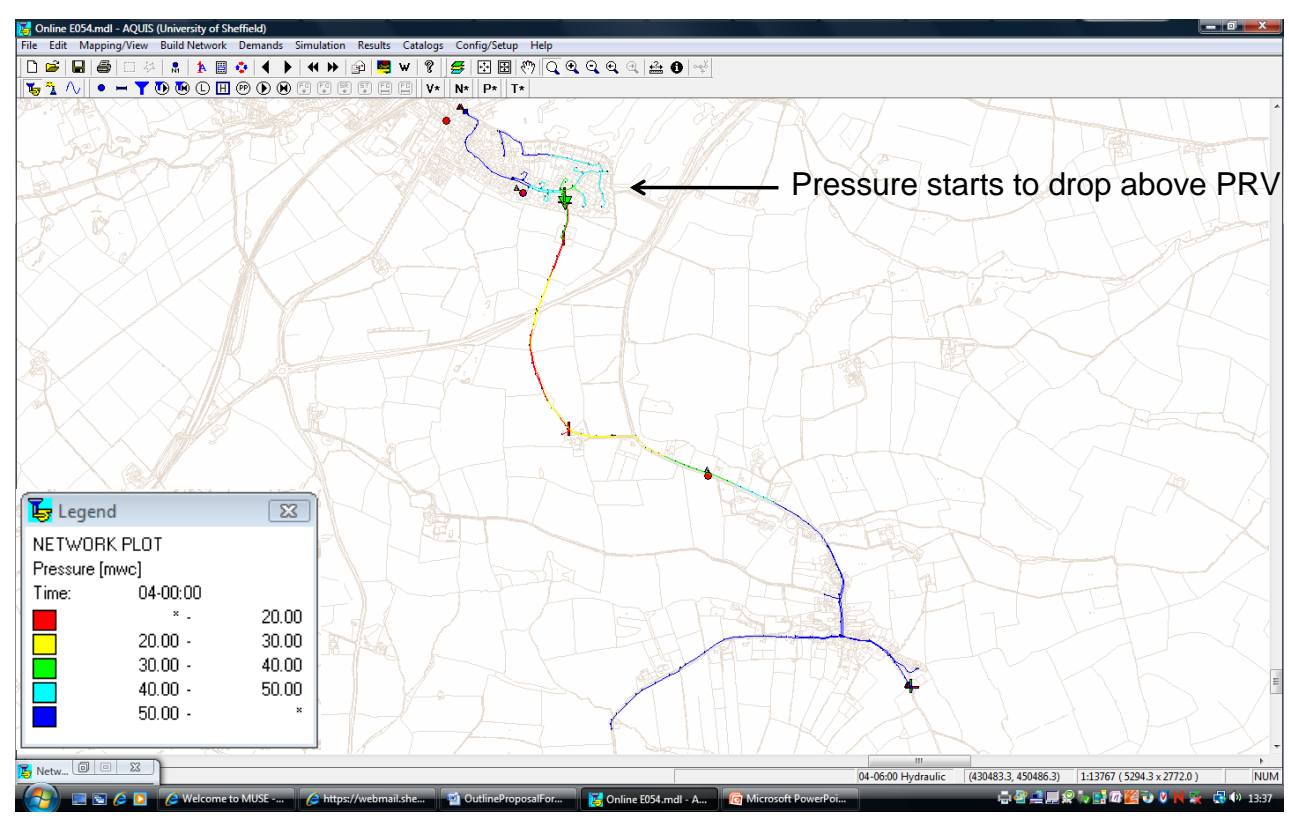

Figure 4. Pressure effect of event at 06:00.

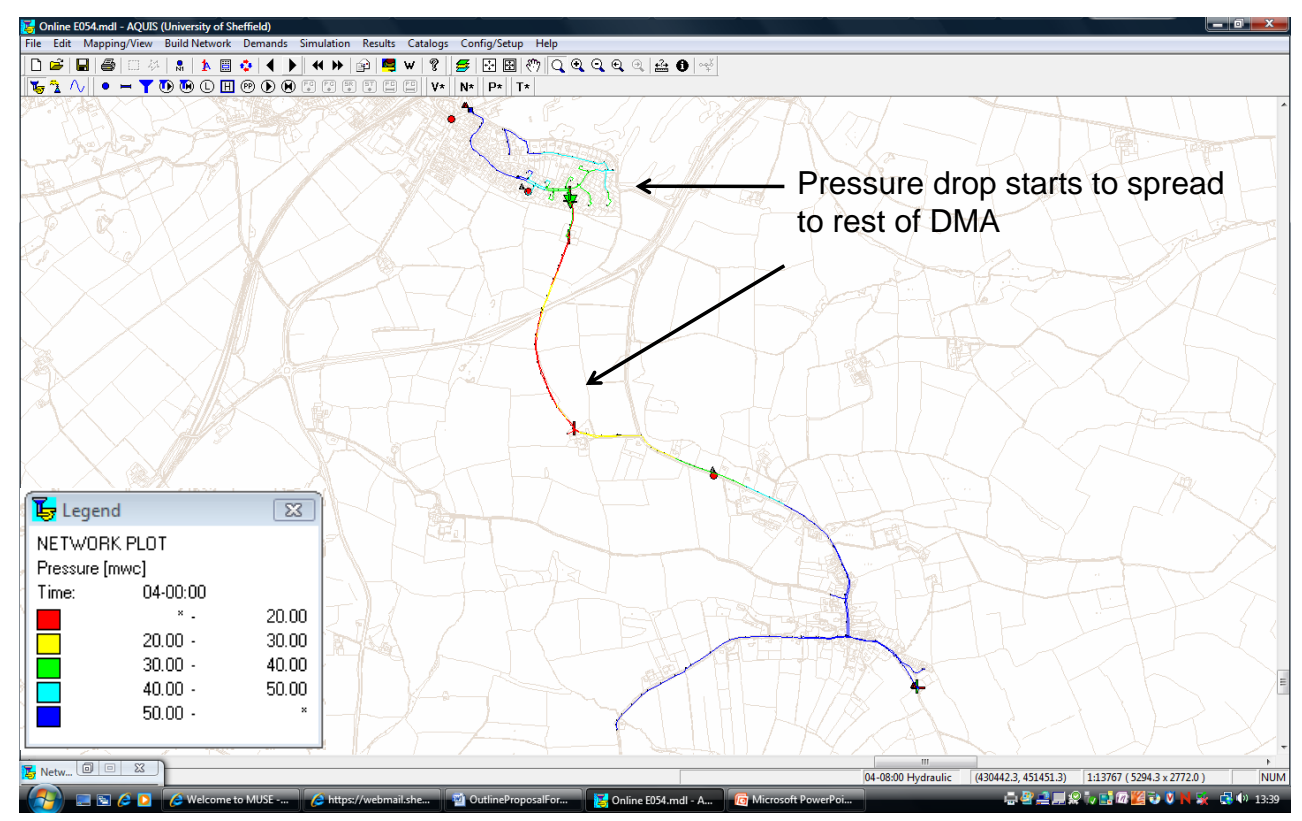

Figure 5. Pressure effect of event at 08:00.

data and not simulated data in which various events in the live distribution system, often unknown to the utility company, will manifest in recorded data.

Following the identification and characterization of the event the online hydraulic model was used in historic mode to test its capability to detect the event by "replaying" the time series data gathered at the time to predict the flow and pressure changes throughout the selected DMA. The online model effectively simulated the flow increases into and out of the DMA from 06:00 and simulated the pressure effect of the event within the DMA. Figures 4 to 6 show the effects of the event propagating through the DMA as simulated by the model.

This sequence of figures illustrates that the on-line model can show the effects of the event on the pressures throughout the network. Each figure is a picture of current network status 


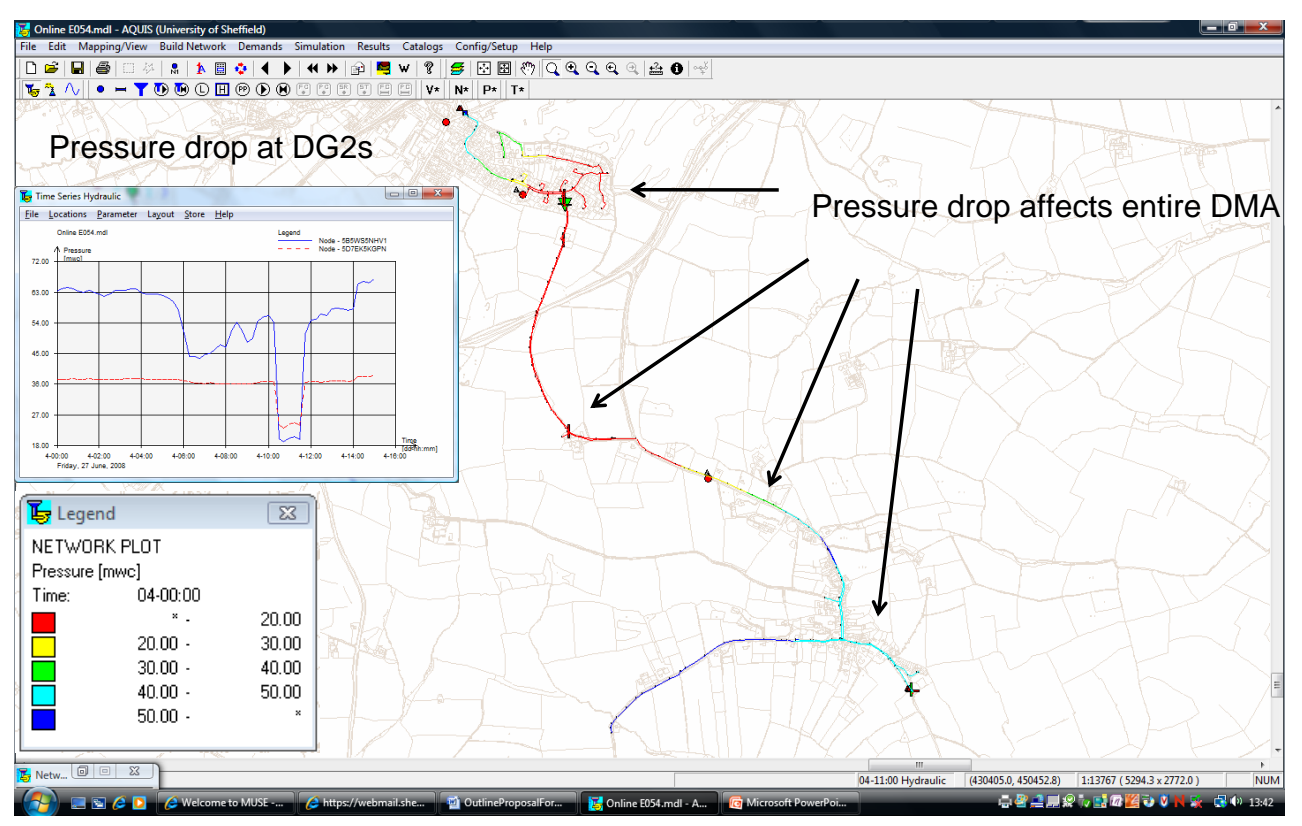

Figure 6. Pressure effect of event at 11:00.

generated using SCADA data specific to the times shown on the slides i.e. Fig. 6 is generated by the model using SCADA data measured at 11:00 imposed as simulation boundary conditions. With the SCADA and modelling system current configuration, the operator would have seen these screen views (operator screens) about an hour after the event. It is perfectly feasible however to gather and transfer data, and carry out the simulation, inside a $15 \mathrm{~min}$ window. As the effects last several hours this would allow sufficient time to warn customers or, if possible, make network changes to limit or even protect customers from the effects. These effects are hydraulic i.e. pressure and flow effects within pipes and even flow reversals. Checks against alarm levels if setup can provide early warning on certain changes for the online model.

The example given in this paper was of a single DMA drawn from a larger network model. Resources would increase proportionately if the model was extended to a wider area, particularly in terms of the instrumentation, communications and computational infrastructure needed. But as this type of modelling moves from the R\&D realm to a "business as usual", the resources required are expected to become more manageable as such technologies continue to improve.

\section{Conclusions}

On-line simulation models require a continuous feed of flow and pressure data from the real network being simulated. Advances in communications technologies have made it viable to transfer such data directly from network instrumentation to the point of use at a frequency suitable to be of practical use. Simulation results presented here have demonstrated that such an on-line model can provide early warning of the magnitude of effect in each pipe in a network and hence on customer service. This capability enables models to simulate and report current network hydraulic state which can be monitored to detect network events such as burst mains and respond to events in a proactive and timely manner.

On-line simulation of a water distribution network provides a tool that can offer tangible and significant operational benefits for system managers. It allows network operators to progress beyond the reactive and develop a more proactive approach to network management. The early effects of a network event such as a burst main can be detected at an early stage which, in turn, can allow network operators to minimize the later effects and sometimes pre-empt and avoid many standards of service failures by manipulating valves, providing an alternative supply or making other appropriate operational change. Pressure effects on every pipe in a network can be captured to gather knowledge about which customers were affected by an event and for how long.

Acknowledgements. This work is part of the NEPTUNE project supported by the UK Science and Engineering Research Council, grant EP/E003192/1, and Industrial Collaborators. The authors would like to particularly acknowledge the assistance of Ridwan Patel from Yorkshire Water Services.

Edited by: I. Worm 


\section{References}

Andersen, J. H. and Powell, R. S.: Simulation of water networking containing controlling elements, J. Water Res. Pl.-ASCE, 125(3), 162-169, 1999.

Bhattacharya, B., Lobbrecht, A. H., and Solomatine, D. P.: Neural networks and reinforcement learning in control of water systems, J. Water Res. Pl.-ASCE, 129(6), 458-465, 2003.

Dandy, G. A., Simpson, A. R., and Murphy, L. J.: An improved genetic algorithm for pipe network optimisation, Water Res., 32(2), 449-458, 1996.

Kapelan, Z., Savic, D. A., and Walters, G. A.: Inverse transient analysis in pipe networks for leakage detection and roughness calibration. Proceedings of Water Network Modelling for Optimal Design and Management CWS 2000, Exeter, UK, 2000.

Koppel, T. and Vassiljev, A.: Calibration of the Model of an Operational Water Distribution System, Proceedings of the IWA World Water Congress (WWC), Vienna, 2008.

Mounce, S. R. and Boxall, J. B.: Implementation of an on-line Artificial Intelligence District Meter Area flow meter data analysis system for abnormality detection: a case study, Proceedings of the 10th IWA conference in instrumentation, control and automation, Cairns, Australia, 2009.
Orr, C., Bouulos, P., Stern, C., and Liu, P.: Developing real-time models of water distribution systems. Modeling and optimisation applications, in: Water Engineering and Management Series, $3-$ 4, edited by: Savic, D. and Walters, G., Vol. 1, Research Studies Pr., 1999.

Powell, R. S., Irving, M. R., Sterling, M. J. H., and Usman, A.: A comparison of three real-time state estimation methods for online monitoring of water distribution systems, in: Computer Applications in Water Supply, Vol. 1, Systems Analysis and Simulation, edited by: Coulbeck, B. and Orr, C. H., 333-348, Research Studies Press Ltd., 1988.

Rao, Z. and Salomons, E.: Development of a real-time, nearoptimal control process for water distribution networks, J. Hydroinform., IWA Publishing, 9(1), 25-37, 2007.

Skipworth, P. J., Saul, A., and Machell, J.: Practical applications of real time hydraulic modelling of water distribution networks, Int. J. COMADEM, 2, 1, 15-21, 1999.

Sterling, M. and Bargiela, A.: Minimum norm state estimation for computer control of water distribution systems, IEE Proceedings Part D, 131(2), 57-63, 1984. 